\title{
Lightlike Submanifolds of a Semi-Riemannian Product Manifold with Quarter Symmetric Non-Metric Connection
}

\author{
Oǧuzhan Bahadır* Erol Kılıç \\ (Communicated by Cihan ÖZGÜR)
}

\begin{abstract}
We study lightlike submanifolds of a semi-Riemannian product manifold. We introduce a class of lightlike submanifolds called screen semi-invariant lightlike submanifold. We consider lightlike submanifolds with respect to a quarter symmetric non-metric connection which is determined by the product structure. We give some equivalent conditions for integrability of distributions with respect to the Levi-Civita connection of semi-Riemannian manifolds and the quarter-symmetric nonmetric connection, and we obtain some results.
\end{abstract}

\section{Introduction}

The theory of degenerate submanifolds of semi-Riemannian manifolds is one of a important topics of diferential geometry because of the following reasons: It is well known that while the geometry of semiRiemannian manifold is fully developed, its counter part of lightlike submanifolds (for which the local geometry is completely different from the non-degenerate case) is relatively new and in a developing stage. Second reason is that the growing importance of null geometry in mathematical physics and limited information on its geometric theory. Several papers have been written on lightlike submanifold in recent years in [3] (see also [4]) by K.L. Duggal and A. Bejancu. Differential Geometry of Lightlike Submanifolds was presented in [9] by K. L. Duggal and B. Sahin. In [5],[6], [7], [8], K. L. Duggal and B. Sahin introduced and studied geometry of classes of lightlike submanifolds in indefinite Kaehler and indefinite Sasakian manifolds which is an umbrella of CR-lightlike, SCR-lightlike, Screen real GCR-lightlie submanifolds. In [2], M. Atceken and E. Kilic introduced semi-invariant lightlike submanifolds of a semi-Riemannian product manifold. In [12], E. Kilic and B. Sahin introduced radical anti-invariant lightlike submanifolds of a semi-Riemannian product manifold and gave some examples and results for lightlike submanifolds. In [13] E. Kilic and O. Bahadir studied lightlike hypersurfaces of a semi-Riemannian product manifold with respect to quarter symmetric non-metric connection.

In [11], Hayden introduced a metric connection with nonzero torsion on a Riemannian manifold. The properties of Riemannian manifolds with semi-symmetric (symmetric) and nonmetric connection have been studied by many authors ([15]-[14]). The idea of quarter-symmetric linear connections in a differential manifold was introduced by Golab [10]. A linear connection is said to be a quarter-symmetric connection if its torsion tensor $\widetilde{T}$ is of the form:

$$
\widetilde{T}(X, Y)=\pi(Y) \varphi X-\pi(X) \varphi Y
$$

for any vector fields $\mathrm{X}, \mathrm{Y}$ on a manifold, where is a $1-$ form and $\varphi$ is a tensor of type $(1,1)$

In this paper, we study $r$ - lightlike and coisotropic submanifolds of a semi- Riemannian product manifold. First, we introduce screen semi-invariant light- like submanifolds of a semi-Riemannian product manifold. We 
define some special distribution of screen semi-invariant lightlike submanifold. Then we give some examples and find their geometric properties of screen semi-invariant lightlike submanifold. Finally, by considering the quarter-symmetric non-metric connection, we study lightlike submanifolds of a semi-Riemannian product manifold. We obtain some results on lightlike submanifolds of a semi- Riemannian product manifold admitting the quarter symmetric non-metric connection.

\section{Preliminaries}

In this section, we give some notations and terminology, given by Duggal and Bejancu in [3] used throughout this paper.

Let $(\widetilde{M}, \widetilde{g})$ be a real $(m+n)$-dimensional semi-Riemannian manifold of constant index such that $1 \leq \nu \leq$ $m+n-1$ and $(M, g)$ be an $m$-dimensional submanifold of $\widetilde{M}$. In case $\widetilde{g}$ is degenerate on the tangent bundle $T M$ of $M, M$ is called a lightlike submanifold of $\widetilde{M}$. Denote by $g$ the induced tensor field of $\widetilde{g}$ on $M$ and suppose $g$ is degenerate. Then, for each tangent space $T_{x} M$ we consider

$$
T_{x} M^{\perp}=\left\{Y_{x} \in T_{x} \widetilde{M} \mid \widetilde{g}_{x}\left(Y_{x}, X_{x}\right)=0, \forall X_{x} \in T_{x} M\right\}
$$

which is a degenerate $n$-dimensional subspace of $T_{x} \widetilde{M}$. Thus, both $T_{x} M$ and $T_{x} M^{\perp}$ are degenerate orthogonal subspaces but no longer complementary subspaces. For this case, there exists a subspace $\operatorname{Rad} T_{x} M=T_{x} M \cap$ $T_{x} M^{\perp}$ called radical (null) subspace. If the mapping

$$
\operatorname{RadTM}: x \in M \longrightarrow \operatorname{Rad}_{x} M
$$

defines a smooth distribution on $M$ of $\operatorname{rank} r>0$, the submanifold $M$ of $\widetilde{M}$ is called $r$-lightlike ( $r$-degenerate) submanifold and RadTM is called the radical (lightlike) distribution on $M$. In the following, there are four possible cases:

Case 1. $M$ is called a $r$-lightlike submanifold if $1 \leq r<\min \{m, n\}$.

Case 2. $M$ is called a coisotropic submanifold if $1<r=n<m$.

Case 3. $M$ is called an isotropic submanifold if $1<r=m<n$.

Case 4. $M$ is called a totally lightlike submanifold if $1<r=m=n$ [3].

In this paper, we have considered case 1 where there exists a non-degenerate screen distribution $S(T M)$ which is a complementary vector subbundle to RadTM in $T M$. Therefore,

$$
T M=\operatorname{Rad} T M \perp s(T M) .
$$

in which $\perp$ denotes orthogonal direct sum. Although $S(T M)$ is not unique, it is canonically isomorphic to the factor vector bundle $T M / \operatorname{RadTM}$. Denote an $r$-lightlike submanifold by $\left(M, g, S(T M), S\left(T M^{\perp}\right)\right)$, where $S\left(T M^{\perp}\right)$ is a complementary vector bundle of $\operatorname{RadTM}$ in $T M^{\perp}$ and $S\left(T M^{\perp}\right)$ is non-degenerate with respect to $\widetilde{g}$. Let us define that $\operatorname{tr}(T M)$ is a complementary (but never orthogonal) vectors bundle to $T M$ in $T \widetilde{M}_{\left.\right|_{M}}$ and

$$
\operatorname{tr} T M=\operatorname{ltr} T M \perp s\left(T M^{\perp}\right),
$$

where $\operatorname{lt}(T M)$ is an arbitrary lightlike transversal vector bundle of $M$. Then we have

$$
\begin{aligned}
T \widetilde{M}_{\left.\right|_{M}} & =T M \oplus \operatorname{tr}(T M) \\
& =(\operatorname{Rad} T M \oplus \operatorname{ltr}(T M)) \perp S(T M) \perp S\left(T M^{\perp}\right)
\end{aligned}
$$

where $\oplus$ denotes direct sum, but it is not orthogonal [3].

The Gauss and Weingarten formulas given by

$$
\begin{aligned}
\widetilde{\nabla}_{X} Y & =\nabla_{X} Y+h(X, Y), \forall X, Y \in \Gamma(T M), \\
\widetilde{\nabla}_{X} V & =-A_{V} X+\nabla_{X}^{t} V, \forall V \in \Gamma(\operatorname{tr} T M),
\end{aligned}
$$

for any $X, Y \in \Gamma(T M)$, where $\left\{\nabla_{X} Y, A_{V} X\right\}$ belong to $\Gamma(T M)$ while $\left\{h(X, Y), \nabla_{X}^{t} V\right\}$ belong to $\Gamma(l \operatorname{tr}(T M))$. 
Suppose $S\left(T M^{\perp}\right) \neq 0$, that is, $M$ is either in Case 1 or in Case 3. According to the decomposition (2.3) we consider the projection morphisms $L$ and $S$ of $\operatorname{tr}(T M)$ on $\operatorname{lt}(T M)$ and $S\left(T M^{\perp}\right)$, respectively. Then $(2.4)-(2.5)$ become

$$
\begin{array}{r}
\widetilde{\nabla}_{X} Y=\nabla_{X} Y+h^{l}(X, Y)+h^{s}(X, Y), \forall X, Y \in \Gamma(T M), \\
\widetilde{\nabla}_{X} N=-A_{N} X+\nabla_{X}^{l} N+D^{s}(X, N), \forall N \in \Gamma(l \operatorname{tr}(T M)), \\
\widetilde{\nabla}_{X} W=-A_{W} X+\nabla_{X}^{s} W+D^{l}(X, W) \forall W \in \Gamma\left(s\left(T M^{\perp}\right)\right) .
\end{array}
$$

where $\quad h^{l}(X, Y)=L h(X, Y), \quad h^{s}(X, Y)=S h(X, Y), \quad\left\{\nabla_{X}^{l} N, D^{l}(X, W)\right\} \in \Gamma(\operatorname{ltr} T M), \quad\left\{\nabla_{X}^{s} W, D^{s}(X, N)\right\} \in$ $\Gamma\left(s\left(T M^{\perp}\right)\right.$ and $\left\{\nabla_{X} Y, A_{N} X, A_{W} X\right\} \in \Gamma(T M)$ [9]. Then, taking account of (2.6)-(2.8) and the Levi-Civita connection $\widetilde{\nabla}$ is a metric, we obtain

$$
\begin{aligned}
\widetilde{g}\left(h^{s}(X, Y), W\right)+g\left(Y, D^{l}(X, W)\right) & =g\left(A_{W} X, Y\right), \\
\widetilde{g}\left(D^{s}(X, N), W\right) & =\widetilde{g}\left(A_{W} X, N\right) .
\end{aligned}
$$

Let $P$ be the projection of $S(T M)$ on $M$. Then according to (2.1) and (2.3) we have

$$
\begin{array}{r}
\nabla_{X} P Y=\nabla_{X}^{*} P Y+h^{*}(X, P Y), \\
\nabla_{X} \xi=-A_{\xi}^{*} X+\nabla_{X}^{* t} \xi,
\end{array}
$$

for any $X, Y \in \Gamma(T M)$ and $\xi \in \Gamma(\operatorname{Rad}(T M))$. By using above equations we obtain

$$
\begin{array}{r}
g\left(h^{l}(X, Y), \xi\right)+g\left(Y, h^{l}(X, \xi)\right)+g\left(Y, \nabla_{X} \xi\right)=0, \\
g\left(h^{*}(X, P Y), N\right)=g\left(A_{N} X, P Y\right), \\
g\left(h^{l}(X, P Y), \xi\right)=g\left(A_{\xi}^{*} X, P Y\right), \\
g\left(A_{N} X, P Y\right)=g\left(N, \widetilde{\nabla}_{X} P Y\right), \\
g\left(h^{l}(X, \xi), \xi\right)=0, A_{\xi}^{*} \xi=0,
\end{array}
$$

In general, the induced connection $\nabla$ on $M$ is not metric connection. Since $\widetilde{\nabla}$ is a metric connection, by using (2.6) we get

$$
\left(\nabla_{X} g\right)(Y, Z)=\widetilde{g}\left(h^{l}(X, Y), Z\right)+\widetilde{g}\left(h^{l}(X, Z), Y\right),
$$

However, it is important to note that $\nabla^{*}$ is a metric connection on $S(T M)$ [9].

\section{Product manifolds}

In this section, we give basic concept for product manifold.

Let $M$ be an $n$-dimensional diferentiable manifold with a tensor field $F$ of type $(1,1)$ on $\widetilde{M}$ such that

$$
F^{2}=I \text {. }
$$

Then $M$ is called an almost product manifold with almost product structure $F$. If we put

$$
\pi=\frac{1}{2}(I+F), \sigma=\frac{1}{2}(I-F)
$$

then we have

$$
\pi+\sigma=I, \pi^{2}=\pi, \sigma^{2}=\sigma, \pi \sigma=\sigma \pi=0, F=\pi-\sigma .
$$

Thus $\pi$ and $\sigma$ define two complementary distributions and $F$ has the eigenvalue of +1 or -1 , If an almost product manifold $\widetilde{M}$ admits a semi-Riemannian metric $\widetilde{g}$ such that

$$
g(F X, F Y)=g(X, Y),
$$

for any $X, Y$ vector fields on $\widetilde{M}$, then $\widetilde{M}$ is called a semi-Riemannian almost product manifold. From (3.1) and (3.2), we have

$$
\widetilde{\nabla}_{X} F Y=F \widetilde{\nabla}_{X} Y
$$

If, for any vector fields $X, Y$ on $\widetilde{M}$,

$$
\widetilde{\nabla} F=0 \text {, that is } \widetilde{\nabla}_{X} F Y=F \widetilde{\nabla}_{X} Y,
$$

then $\widetilde{M}$ is called a semi-Riemannian product manifold, where $\widetilde{\nabla}$ is the Levi-Civita connection on $\widetilde{M}$ [16]. 


\section{Lightlike Submanifolds of Semi-Riemannian Product Manifolds}

In this section we defined screen semi-invariant lightlike submanifolds of a semi-Riemannian product manifold. We give some examples and study their geometric properties.

Let $\left(M, g, S(T M), S\left(T M^{\perp}\right)\right)$ be a lightlike submanifold of a semi-Riemannian product manifold $(\widetilde{M}, \widetilde{g}, F)$. For each $X$ tangent to $M, F X$ can be written as follows:

$$
F X=f X+w X
$$

where $f X$ and $w X$ are the tangential and the transversal parts of $F X$, respectively. In addition, for any $V \in \Gamma(\operatorname{tr}(T M)), F V$ can be written as:

$$
F V=B V+C V,
$$

where $B V$ and $C V$ are the tangential and the transversal parts of $F V$, respectively .

Definition 4.1. Let $\left(M, g, S(T M), S\left(T M^{\perp}\right)\right)$ be a lightlike submanifold of a semi-Riemannian product manifold $(\widetilde{M}, \widetilde{g}, F)$. If $F \operatorname{Rad} T M \subset s(T M), F l t r T M \subset s(T M)$ and $F s\left(T M^{\perp}\right) \subset s(T M)$ then we say that $M$ is a screen semi-invariant lightlike submanifold.

Now, let $M$ be a screen semi-invariant lightlike submanifold of a semi-Riemannian product manifold. If we set $L_{1}=F \operatorname{RadTM}, L_{2}=F \operatorname{lt} T M$ and $L_{3}=F s\left(T M^{\perp}\right)$ then we can write

$$
s(T M)=L_{0} \perp\left\{L_{1} \oplus L_{2}\right\} \perp L_{3},
$$

where $L_{0}$ is a $(m-r-4)$ - dimensional distribution. Hence we have the following decomposition:

$$
\begin{aligned}
T M & =\left\{L_{1} \oplus L_{2}\right\} \perp L_{3} \perp L_{0} \perp \operatorname{RadTM}, \\
T \widetilde{M} & =\left\{L_{1} \oplus L_{2}\right\} \perp L_{3} \perp L_{0} \perp\{\operatorname{RadTM} \oplus \operatorname{ltr} T M\} .
\end{aligned}
$$

According to this definition we can write

$$
L=L_{0} \perp L_{1} \perp \operatorname{RadTM}
$$

and

$$
L^{\prime}=L_{2} \perp L_{3}
$$

Thus we have

$$
T M=L \oplus L^{\prime}
$$

From (4.7) and (4.8) we have the following proposition

Proposition 4.1. Let $\left(M, g, S(T M), S\left(T M^{\perp}\right)\right)$ be a screen semi-invariant lightlike submanifold of a semi-Riemannian product manifold $(\widetilde{M}, \widetilde{g}, F)$. Then $L$ and $L^{\prime}$ are $F$-invariant and anti-invariant distributions, respectively.

For Case (2), we know that $s\left(T M^{\perp}\right)=\{0\}$. Therefore we have the following decomposition

$$
\begin{aligned}
s(T M) & =\left\{L_{1} \oplus L_{2}\right\} \perp L_{0}, \\
T M & =\left\{L_{1} \oplus L_{2}\right\} \perp L_{0} \perp \operatorname{RadTM}, \\
T \widetilde{M} & =\left\{L_{1} \oplus L_{2}\right\} \perp L_{0} \perp\{\operatorname{RadTM} \oplus \operatorname{ltr} T M\}, \\
T M & =L \oplus L_{2} .
\end{aligned}
$$

Proposition 4.2. Let $\left(M, g, S(T M), S\left(T M^{\perp}\right)\right)$ be a screen semi-invariant lightlike submanifold of a semi-Riemannian product manifold $(\widetilde{M}, \widetilde{g}, F)$. The distribution $L_{0}$ is a invariant distribution with respect to $F$.

Example 4.1. Let $\mathrm{M}$ be a submanifold of semi- Riemannian product manifold $\widetilde{M}=R_{2}^{5} \times R_{1}^{3}$ with metric tensor $\widetilde{g}=\pi^{*} g_{1}+\sigma^{*} g_{2}$, where $g_{1}$ and $g_{2}$ are standard metric tensors of $R_{2}^{5}$ and $R_{1}^{3}$, respectively. Consider the 
submanifold $M$ in $\widetilde{M}$ defined by the equations:

$$
\begin{aligned}
& x_{1}=t_{1}+t_{2}+2 t_{3}+2 t_{4}+(1+2 \sqrt{2}) t_{5}-3 t_{6}, \\
& x_{2}=2 t_{1}+2 t_{2}+t_{3}+(2-\sqrt{2}) t_{5}+(2-\sqrt{2}) t_{6}, \\
& x_{3}=t_{3}+t_{4}+3 \sqrt{2} t_{5}-6 t_{6}, \\
& x_{4}=t_{1}+t_{2}+2 t_{3}+\sqrt{2} t_{4}+t_{5}+t_{6}, \\
& x_{5}=2 t_{1}+2 t_{2}+t_{3}+t_{4}+2 t_{5}-\sqrt{2} t_{6}, \\
& x_{6}=\sqrt{2} t_{1}-\sqrt{2} t_{2}-\sqrt{2} t_{3}-3 t_{4}, \\
& x_{7}=-\sqrt{2} t_{3}-t_{4}, \\
& x_{8}=2 t_{1}-2 t_{2}-t_{3}-2 \sqrt{2} t_{4},
\end{aligned}
$$

where $t_{i}, 1 \leq i \leq 4$, are real parameters. Then the tangent bundle $T M$ is spanned by $\left\{U_{1}, U_{2}, U_{3}, U_{4}, U_{5}, U_{6}\right\}$, where

$$
\begin{aligned}
U_{1} & =\frac{\partial}{\partial x_{1}}+2 \frac{\partial}{\partial x_{2}}+\frac{\partial}{\partial x_{4}}+2 \frac{\partial}{\partial x_{5}}+\sqrt{2} \frac{\partial}{\partial x_{6}}+2 \frac{\partial}{\partial x_{8}}, \\
U_{2} & =\frac{\partial}{\partial x_{1}}+2 \frac{\partial}{\partial x_{2}}+\frac{\partial}{\partial x_{4}}+2 \frac{\partial}{\partial x_{5}}-\sqrt{2} \frac{\partial}{\partial x_{6}}-2 \frac{\partial}{\partial x_{8}}, \\
U_{3} & =2 \frac{\partial}{\partial x_{1}}+\frac{\partial}{\partial x_{2}}+\frac{\partial}{\partial x_{3}}+2 \frac{\partial}{\partial x_{4}}+\frac{\partial}{\partial x_{5}}-\sqrt{2} \frac{\partial}{\partial x_{6}}-\sqrt{2} \frac{\partial}{\partial x_{7}}-\frac{\partial}{\partial x_{8}}, \\
U_{4} & =2 \frac{\partial}{\partial x_{1}}+\frac{\partial}{\partial x_{3}}+\sqrt{2} \frac{\partial}{\partial x_{4}}+\frac{\partial}{\partial x_{5}}-3 \frac{\partial}{\partial x_{6}}-\frac{\partial}{\partial x_{7}}-2 \sqrt{2} \frac{\partial}{\partial x_{8}}, \\
U_{5} & =(1+2 \sqrt{2}) \frac{\partial}{\partial x_{1}}+(2-\sqrt{2}) \frac{\partial}{\partial x_{2}}+3 \sqrt{2} \frac{\partial}{\partial x_{3}}+\frac{\partial}{\partial x_{4}}+2 \frac{\partial}{\partial x_{5}}, \\
U_{6} & =-3 \frac{\partial}{\partial x_{1}}+(2-\sqrt{2}) \frac{\partial}{\partial x_{2}}-6 \frac{\partial}{\partial x_{3}}+\frac{\partial}{\partial x_{4}}-\sqrt{2} \frac{\partial}{\partial x_{5}} .
\end{aligned}
$$

It is easy to check that $M$ is a lightlike submanifold and $U_{1}$ is a degenerate vector. Then we have $\operatorname{Rad} T M=$ $\operatorname{Span}\left\{U_{1}\right\}$ and $S(T M)=\operatorname{Span}\left\{U_{2}, U_{3}, U_{4}, U_{5}, U_{6}\right\}$. By direct calculations we obtain

$$
\operatorname{ltrTM}=\operatorname{Span}\left\{N=2 \frac{\partial}{\partial x_{1}}+\frac{\partial}{\partial x_{3}}+\sqrt{2} \frac{\partial}{\partial x_{4}}+\frac{\partial}{\partial x_{5}}+3 \frac{\partial}{\partial x_{6}}+\frac{\partial}{\partial x_{7}}+2 \sqrt{2} \frac{\partial}{\partial x_{8}}\right\},
$$

and

$$
s\left(T M^{\perp}\right)=\operatorname{Span}\left\{u=2 \frac{\partial}{\partial x_{1}}+\frac{\partial}{\partial x_{2}}+\frac{\partial}{\partial x_{3}}+2 \frac{\partial}{\partial x_{4}}+\frac{\partial}{\partial x_{5}}+\sqrt{2} \frac{\partial}{\partial x_{6}}+\sqrt{2} \frac{\partial}{\partial x_{7}}+\frac{\partial}{\partial x_{8}}\right\} .
$$

Hence $M$ is a 6 - dimensional 1 - lightlike submanifold. Furthermore, we get

$$
\begin{aligned}
& F \xi=U_{2} \in \Gamma(s(T M)), F N=U_{4} \in \Gamma(s(T M)), \\
& F u=U_{3} \in \Gamma(s(T M)), F U_{5}=U_{5}, F U_{6}=U_{6} .
\end{aligned}
$$

Thus we have

$$
\begin{gathered}
L_{0}=\operatorname{Span}\left\{U_{5}, U_{6}\right\}, L_{1}=\operatorname{Span}\left\{U_{2}\right\}, \\
L_{2}=\operatorname{Span}\left\{U_{4}\right\}, L_{3}=\operatorname{Span}\left\{U_{3}\right\} .
\end{gathered}
$$

Therefore $M$ is screen semi-invariant lightlike submanifold of $\widetilde{M}$.

Example 4.2. Let $\left(\widetilde{M}=R_{2}^{5}, \widetilde{g}\right)$ be a 5-dimensional semi-Euclidean space with signature $(-,-,+,+,+)$ and $\left(x_{1}, x_{2}, x_{3}, x_{4}, x_{5}\right)$ be the standard coordinate system of $R_{2}^{5}$. If we set

$$
F\left(x_{1}, x_{2}, x_{3}, x_{4}, x_{5}\right)=\left(x_{2}, x_{1}, x_{4}, x_{3}, x_{5}\right),
$$

then $F^{2}=I$ and $F$ is a product structure on $R_{2}^{5}$. Consider a submanifold $M$ in $\widetilde{M}$ by the equations:

$$
\begin{aligned}
& x_{1}=2 t_{1}+t_{2}+t_{3}-\arcsin _{4}, \\
& x_{2}=t_{1}+2 t_{2}+2 t_{3}+\operatorname{arcsint}_{4}, \\
& x_{3}=-t_{2}+t_{3}+2 \operatorname{arcsint}_{4}, \\
& x_{4}=-t_{1}, \\
& x_{5}=2 t_{1}+2 t_{2}+2 t_{3}-\frac{1}{2} \operatorname{arcsint}_{4} .
\end{aligned}
$$


where $t_{i}, 1 \leq i \leq 4$, are real parameters. Then the tangent bundle $T M$ is spanned by $\left\{U_{1}, U_{2}, U_{3}, U_{4}\right\}$, where

$$
\begin{aligned}
U_{1} & =2 \frac{\partial}{\partial x_{1}}+\frac{\partial}{\partial x_{2}}-\frac{\partial}{\partial x_{4}}+2 \frac{\partial}{\partial x_{5}}, \\
U_{2} & =\frac{\partial}{\partial x_{1}}+2 \frac{\partial}{\partial x_{2}}-\frac{\partial}{\partial x_{3}}+2 \frac{\partial}{\partial x_{5}}, \\
U_{3} & =\frac{\partial}{\partial x_{1}}+2 \frac{\partial}{\partial x_{2}}+\frac{\partial}{\partial x_{3}}+2 \frac{\partial}{\partial x_{5}}, \\
U_{4} & =-\frac{\partial}{\partial x_{1}}+\frac{\partial}{\partial x_{2}}+2 \frac{\partial}{\partial x_{3}}-\frac{1}{2} \frac{\partial}{\partial x_{5}},
\end{aligned}
$$

It is easy to check that $M$ is a $1-$ lightlike submanifold and $U_{1}$ is a degenerate vector. Then we have $\operatorname{RadTM}=\operatorname{Span}\{\xi\}$ and $S(T M)=\operatorname{Span}\left\{U_{2}, U_{3}, U_{4}\right\}$. Then by direct calculations we get

$$
\operatorname{ltr} T M=\operatorname{Sp}\left\{N=2 \frac{\partial}{\partial x_{1}}+\frac{\partial}{\partial x_{2}}+\frac{\partial}{\partial x_{4}}+2 \frac{\partial}{\partial x_{5}}\right\} .
$$

Moreover we can write $L_{0}=S p\left\{U_{4}\right\}, L_{1}=S p\left\{U_{2}\right\}, L_{2}=S p\left\{U_{3}\right\}$. Thus $M$ is a screen semi-invariant coisotropic submanifold of $\widetilde{M}$.

Now, let $\left(M, g, S(T M), S\left(T M^{\perp}\right)\right)$ be a screen semi-invariant lightlike submanifold of a semi-Riemannian product manifold $(\widetilde{M}, \widetilde{g}, F)$. For any $X, Y \in \Gamma(T M)$ we obtain

$$
\widetilde{\nabla}_{X} F Y=\nabla_{X} f Y+h(X, f Y)-A_{w Y} X+\nabla_{X}^{t} w Y
$$

and

$$
F \widetilde{\nabla}_{X} Y=f \nabla_{X} Y+w \nabla_{X} Y+B h(X, Y) .
$$

Since $F$ is parallel on $M$, from (4.13) and (4.14) we get

$$
\begin{aligned}
\left(\nabla_{X} f\right) Y & =A_{w Y} X+B h(X, Y), \\
w \nabla_{X} Y & =\nabla_{X}^{t} w Y+h(X, f Y) .
\end{aligned}
$$

Theorem 4.1. Let $\left(M, g, S(T M), S\left(T M^{\perp}\right)\right)$ be a screen semi-invariant $r$-lightlike submanifold of a semi-Riemannian product manifold $(\widetilde{M}, \widetilde{g}, F)$. Then the following assertions are equivalent:

(i) $M$ is mixed geodesic.

(ii) $A_{\xi}^{*} X$ and $A_{W} X$ have no component in $L_{1}$ and $L_{3}$, for any $X \in \Gamma(L)$.

(iii) $A_{F Y} X$ has no component $L_{2}$ and $L_{3}$, for any $X \in \Gamma(L)$ and $Y \in \Gamma\left(L^{\prime}\right)$.

Proof. $M$ is mixed geodesic if and only if

$$
g(h(X, Y), \xi)=0 \text { and } g(h(X, Y), W)=0,
$$

for any $N \in \Gamma(\operatorname{ltr} T M), W \in \Gamma\left(s\left(T M^{\perp}\right)\right), X \in \Gamma(L)$ and $Y \in \Gamma\left(L^{\prime}\right)$. From (2.4), (2.7), (2.8) and (2.12) we obtain

$$
g(h(X, Y), \xi)=g\left(Y, A_{\xi}^{*} X\right)
$$

and

$$
g(h(X, Y), W)=g\left(Y, A_{W} X\right) .
$$

Thus we get $(i) \Leftrightarrow(i i)$. Similarly from (2.4), (2.5), (3.3) and (3.4) we obtain

$$
g(h(X, Y), \xi)=-g\left(A_{F Y} X, F \xi\right)
$$

and

$$
g(h(X, Y), W)=-g\left(A_{F Y} X, F W\right) .
$$

Therefore we conclude $(i) \Leftrightarrow($ iii $)$. Thus we have our assertion

If $M$ is coisotropic submanifold, that is $S\left(T M^{\perp}\right)=0$, then from (2.11) we have the following theorem. 
Theorem 4.2. Let $(M, g, S(T M))$ be a screen semi-invariant coisotropic submanifold of a semi-Riemannian product manifold $(\widetilde{M}, \widetilde{g}, F)$. Then the following assertions are equivalent:

(i) $M$ is mixed geodesic.

(ii) $A_{\xi}^{*} X$ have no component in $L_{1}$, for any $X \in \Gamma(L)$.

(iii) $h^{*}(X, F \xi)=0$, for any $X \in \Gamma(L)$.

Theorem 4.3. Let $\left(M, g, S(T M), S\left(T M^{\perp}\right)\right)$ be a screen semi-invariant $r$-lightlike submanifold of a semi-Riemannian product manifold $(\widetilde{M}, \widetilde{g}, F)$. Then the following assertions are equivalent:

(i) $M$ is $L$-geodesic.

(ii) $A_{\xi}^{*} X \in \Gamma\left(L_{1} \perp L_{3}\right)$ and $g\left(Y, A_{W} X\right)=g\left(Y, D^{l}(X, W)\right)$, for any $X, Y \in \Gamma(L)$

(iii) $\nabla_{X} F Y$ have no component in $L_{2}$ and $L_{3}$, for any $X, Y \in \Gamma(L)$.

Proof. It is known that $M$ is $L$ - geodesic if and only if

$$
g(h(X, Y), \xi)=0 \text { and } g(h(X, Y), W)=0,
$$

for any $X, Y \in \Gamma(L), N \in \Gamma($ ltr $T M), W \in \Gamma\left(s\left(T M^{\perp}\right)\right)$. Accordingly from (2.4), (2.7), (2.8) and (2.12) we obtain

$$
g(h(X, Y), \xi)=g\left(Y, A_{\xi}^{*} X\right)
$$

and

$$
g(h(X, Y), W)=g\left(Y, A_{W} X-D^{l}(X, W)\right) .
$$

Thus we get $(i) \Leftrightarrow(i i)$. Similarly from (2.4), (2.5), (3.3) and (3.4) we have

$$
\left.g(h(X, Y), \xi)=-g\left(\nabla_{X} F Y, F \xi\right)\right)
$$

and

$$
\left.g(h(X, Y), W)=-g\left(\nabla_{X} F Y, F W\right)\right) .
$$

This is $(i) \Leftrightarrow(i i i)$.

From above the theorem, we have the following theorem.

Theorem 4.4. Let $(M, g, S(T M))$ be a screen semi-invariant coisotropic submanifold of a semi-Riemannian product manifold $(\widetilde{M}, \widetilde{g}, F)$. Then the following assertions are equivalent:

(i) $M$ is $L$-geodesic.

(ii) $A_{\xi}^{*} X \in \Gamma\left(L_{1}\right)$, for any $X \in \Gamma(L)$.

(iii) $\nabla_{X}^{*} F \xi \in \Gamma\left(L_{1}\right)$, for any $X \in \Gamma(L)$.

Theorem 4.5. Let $\left(M, g, S(T M), S\left(T M^{\perp}\right)\right)$ be a screen semi-invariant $r$-lightlike submanifold of a semi-Riemannian product manifold $(\widetilde{M}, \widetilde{g}, F)$. Then the following assertions are equivalent:

(i) $M, L^{\prime}-$ geodeziktir.

(ii) $A_{W} X$ and $A_{\xi}^{*} X$ have no component in $L_{1}$ and $L_{3}$, for any $X \in \Gamma\left(L^{\prime}\right)$.

(iii) $A_{F Y} X$ have no component in $L_{2}$ and $L_{3}$, for any $X, Y \in \Gamma\left(L^{\prime}\right)$.

Proof. For any $N \in \Gamma(\operatorname{ltr} T M), W \in \Gamma\left(s\left(T M^{\perp}\right)\right)$ and $X, Y \in \Gamma(L)$, by using (2.12) and Gauss- Weingarten formulas we obtain

$$
g(h(X, Y), \xi)=g\left(Y, A_{\xi}^{*} X\right),
$$

and

$$
g(h(X, Y), W)=g\left(Y, A_{W} X\right) .
$$

Thus we get $(i) \Leftrightarrow(i i)$. Moreover from (3.2), (3.3) and Gauss-Weingarten formulas we get

$$
g(h(X, Y), \xi)=-g\left(A_{F Y} X, F \xi\right)
$$

and

$$
g(h(X, Y), W)=-g\left(A_{F Y} X, F W\right) .
$$

This is $(i) \Leftrightarrow(i i i)$. 
Theorem 4.6. Let $\left(M, g, S(T M), S\left(T M^{\perp}\right)\right)$ be a screen semi-invariant $r$-lightlike submanifold of a semi-Riemannian product manifold $(\widetilde{M}, \widetilde{g}, F)$. Then the following assertions are equivalent:

(i) $L$ is parallel distribution.

(ii) $h(X, F Y)=0, X, Y \in \Gamma(L)$.

(iii) $\left(\nabla_{X} f\right) Y=0$, for any $X, Y \in \Gamma(L)$.

Proof. For any $N \in \Gamma(\operatorname{lt} T M), W \in \Gamma\left(s\left(T M^{\perp}\right)\right)$ and $X, Y \in \Gamma(L)$ by using Gauss-Weingarten formulas we have

$$
g\left(\nabla_{X} Y, F \xi\right)=-g\left(h^{l}(X, F Y), \xi\right)
$$

and

$$
g\left(\nabla_{X} Y, F W\right)=-g\left(h^{s}(X, F Y), W\right)
$$

Thus we get $(i) \Leftrightarrow(i i)$. Since $F$ is parallel, from (2.4), (4.1) we obtain

$$
h(X, F Y)=-\left(\nabla_{X} f\right) Y+w \nabla_{X} Y+F h(X, Y)
$$

If we take tangential and transversal parts of this last equation we have

$$
\left(\nabla_{X} f\right) Y=F h(X, Y)
$$

Thus we get $(i i) \Leftrightarrow(i i i)$.

Proposition 4.3. Let $\left(M, g, S(T M), S\left(T M^{\perp}\right)\right)$ be a screen semi-invariant r-lightlike submanifold of a semi-Riemannian product manifold $(\widetilde{M}, \widetilde{g}, F)$. Then the distribution $L^{\prime}$ is parallel on $M$ if and only if $A_{F Y} X \in \Gamma\left(L^{\prime}\right)$.

Proof. For any $X \in \Gamma(T M), Y \in \Gamma\left(L^{\prime}\right)$ and $Z \in \Gamma\left(L_{0}\right)$, from (3.2), (3.3) and Gauss-Weingarten formulas we obtain

$$
\begin{aligned}
g\left(\nabla_{X} Y, N\right) & =g\left(\widetilde{\nabla}_{X} Y, N\right)=g\left(\widetilde{\nabla}_{X} F Y, F N\right)=-g\left(A_{F Y} X, F N\right), \\
g\left(\nabla_{X} Y, F N\right) & =g\left(\widetilde{\nabla}_{X} F Y, N\right)=-g\left(A_{F Y} X, N\right), \\
g\left(\nabla_{X} Y, Z\right) & =g\left(\widetilde{\nabla}_{X} Y, Z\right)=g\left(\widetilde{\nabla}_{X} F Y, F Z\right)=g\left(A_{F Y} X, F Z\right),
\end{aligned}
$$

From (4.17), (4.18) and (4.19), proof is completed.

Theorem 4.7. Let $\left(M, g, S(T M), S\left(T M^{\perp}\right)\right)$ be a screen semi-invariant r-lightlike submanifold of a semi-Riemannian product manifold $(\widetilde{M}, \widetilde{g}, F)$. Then the following assertions are equivalent:

(i) $L$ is integrable.

(ii) $h(X, F Y)=h(Y, F X), X, Y \in \Gamma(L)$.

(iii) $\left(\nabla_{X} f\right) Y=\left(\nabla_{Y} f\right) X, X, Y \in \Gamma(L)$.

Proof. For any $X, Y \in \Gamma(L)$, from (2.4) we obtain

$$
g([X, Y], F \xi)=g(h(X, F Y)-h(Y, F X), \xi) .
$$

Thus we get $(i) \Leftrightarrow(i i)$. From (2.4) and (4.1) we obtain

$$
h(X, F Y)=F \nabla_{X} Y+F h(X, Y)-\nabla_{X} F Y .
$$

Interchanging role of $X$ and $Y$ we have

$$
h(Y, F X)=F \nabla_{Y} X+F h(Y, X)-\nabla_{Y} F X .
$$

From (4.20) and (4.21) we get

$$
h(X, F Y)-h(Y, F X)=-\left(\nabla_{X} f\right) Y+\left(\nabla_{Y} f\right) X+w([X, Y])
$$

If we take tangential and transversal parts of this last equation we have $($ ii $) \Leftrightarrow($ iii $)$. 
Proposition 4.4. Let $\left(M, g, S(T M), S\left(T M^{\perp}\right)\right)$ be a screen semi-invariant r-lightlike submanifold of a semi-Riemannian product manifold $(\widetilde{M}, \widetilde{g}, F)$. Then the distribution $L^{\prime}$ is integrable if and only if

$$
A_{F X} Y=A_{F Y} X, X, Y \in \Gamma\left(L^{\prime}\right),
$$

Proof. For any $N \in \Gamma(\operatorname{ltr} T M), Z \in \Gamma\left(L_{0}\right)$ and $X, Y \in \Gamma\left(L^{\prime}\right)$, from (2.5) we obtain

$$
\begin{aligned}
& g([X, Y], F N)=g\left(A_{F X} Y-A_{F Y} X, N\right), \\
& g([X, Y], Z)=g\left(A_{F X} Y-A_{F Y} X, F Z\right) .
\end{aligned}
$$

nnd

$$
g([X, Y], Z)=g\left(A_{F X} Y-A_{F Y} X, F Z\right) .
$$

From (4.22) and (4.24) we get the proof.

Theorem 4.8. Let $\left(M, g, S(T M), S\left(T M^{\perp}\right)\right)$ be a screen semi-invariant $r$-lightlike submanifold of a semi-Riemannian product manifold $(\widetilde{M}, \widetilde{g}, F)$. Then $M$ is a locally product manifold according to the decomposition (4.8) if and only if $f$ is parallel with respect to induced connection $\nabla$, that is $\nabla f=0$.

Proof. Let $M$ be a locally product manifold. Then the leaves of distributions $L$ and $L^{\prime}$ are both totally geodesic in $M$. Since the distribution $L$ is invariant with respect to $F$ then, for any $Y \in \Gamma(L), F Y \in \Gamma(L)$. Thus $\nabla_{X} Y$ and $\nabla_{X} f Y$ belong to $\Gamma(L)$ We know that $\widetilde{\nabla} F=0$. Then from the Gauss formula we obtain

$$
\nabla_{X} f Y+h(X, f Y)=f \nabla_{X} Y+w \nabla_{X} Y+F h(X, Y) .
$$

Comparing the tangential and normal parts with respect to $L$ of (4.25), we have

$$
\left(\nabla_{X} f\right) Y=0 .
$$

For any $X \in \Gamma(T M)$ and $Z \in \Gamma\left(L^{\prime}\right)$, since $f Z=0$, we get $\nabla_{X} f Z=0$ and $f \nabla_{X} Z=0$, that is $\left(\nabla_{X} f\right) Z=0$. Thus we have $\nabla f=0$ on $M$.

Conversely, we assume that $\nabla f=0$ on $M$. Then we have $\nabla_{X} f Y=f \nabla_{X} Y$, for any $X, Y \in \Gamma(L)$ and $\nabla_{W} f U=$ $f \nabla_{W} U=0$, for any $U, W \in \Gamma\left(L^{\prime}\right)$. Hence, the leaves of the distributions $L$ and $L^{\prime}$ are totally geodesic in $M$.

\section{Lightlike Submanifolds of Semi-Riemannian Product Manifolds with Quarter Symmetric Non-Metric Connection}

In this section we investigated screen semi-invariant lightlike submanifolds of a semi-Riemannian product manifold with quarter symmetric non-metric connection and we study their geometric properties.

Let $(M, g, F)$ be a semi-Riemannian product manifold and $\widetilde{\nabla}$ be the Levi-Civita connection on $M$. If we set

$$
\widetilde{D}_{X} Y=\widetilde{\nabla}_{X} Y+\pi(Y) F X
$$

for any $X, Y \in \Gamma(T \widetilde{M})$, then $\widetilde{D}$ is a linear connection on $\widetilde{M}$, where $u$ is a 1-form on $\widetilde{M}$ with $U$ as associated vector field, that is

$$
\pi(X)=\widetilde{g}(X, U) .
$$

The torsion tensor of $\widetilde{D}$ on $\widetilde{M}$ denoted by $\widetilde{T}$. Then we obtain

$$
\widetilde{T}(X, Y)=\pi(Y) F X-\pi(X) F Y,
$$

and

$$
\left(\widetilde{D}_{X} \widetilde{g}\right)(Y, Z)=-\pi(Y) \widetilde{g}(F X, Z)-\pi(Z) \widetilde{g}(F X, Y),
$$

for any $X, Y \in \Gamma(T \widetilde{M})$. Thus $\widetilde{D}$ is a quarter-symmetric non-metric connection on $\widetilde{M}$. From (5.1) we have

$$
\left(\widetilde{D}_{X} F\right) Y=\pi(F Y) F X-\pi(Y) X .
$$


Replacing $X$ by $F X$ and $Y$ by $F Y$ in (5.4) we obtain

$$
\left(\widetilde{D}_{F X} F\right) F Y=\pi(Y) X-\pi(F Y) F X .
$$

Thus we have

$$
\left(\widetilde{D}_{X} F\right) Y+\left(\bar{D}_{F X} F\right) F Y=0
$$

If we set

$$
{ }^{\prime} F(X, Y)=\widetilde{g}(F X, Y)
$$

for any $X, Y \in \Gamma(T \bar{M})$, from (5.1) we get

$$
\left(\widetilde{D}_{X}{ }^{\prime} F\right)(Y, Z)=\left(\widetilde{\nabla}_{X}{ }^{\prime} F\right)(Y, Z)-\pi(Y) \widetilde{g}(X, Z)-\pi(Z) \widetilde{g}(X, Y) .
$$

From (5.1) the curvature tensor $\widetilde{R}^{D}$ of the quarter-symmetric non-metric connection $\widetilde{D}$ is given by

$$
\widetilde{R}^{D}(X, Y) Z=\widetilde{R}(X, Y) Z+\widetilde{\lambda}(X, Z) F Y-\widetilde{\lambda}(Y, Z) F X,
$$

for any $X, Y, Z \in \Gamma(T \widetilde{M})$, where $\widetilde{\lambda}$ is a $(0,2)$-tensor given by $\widetilde{\lambda}(X, Z)=\left(\widetilde{\nabla}_{X} \pi\right)(Z)-\pi(Z) \pi(F X)$. If we set $\widetilde{R}^{D}(X, Y, Z, W)=\widetilde{g}\left(\bar{R}^{D}(X, Y) Z, W\right)$, then, from (5.9), we obtain

$$
\widetilde{R}^{D}(X, Y, Z, W)=-\widetilde{R}^{D}(Y, X, Z, W) .
$$

We note that the Riemannian curvature tensor $\widetilde{R}^{D}$ of $\widetilde{D}$ does not satisfy the other curvature-like properties. But, from (5.9), we have

$$
\begin{aligned}
\widetilde{R}^{D}(X, Y) Z+\widetilde{R}^{D}(Y, Z) X+\widetilde{R}^{D}(Z, X) Y & =(\widetilde{\lambda}(Z, Y)-\widetilde{\lambda}(Y, Z)) F X \\
& +(\widetilde{\lambda}(X, Z)-\widetilde{\lambda}(Z, X)) F Y \\
& +(\widetilde{\lambda}(Y, X)-\widetilde{\lambda}(X, Y)) F Z .
\end{aligned}
$$

Thus we have the following proposition.

Proposition 5.1. Let $\left(M, g, S(T M), S\left(T M^{\perp}\right)\right)$ be a lightlike submanifold of a semi-Riemannian product manifold $(\widetilde{M}, \widetilde{g})$. Then the first Bianchi identity of the quarter symmetric non-metric connection $\widetilde{D}$ on $M$ is provided if and only if $\widetilde{\lambda}$ is symmetric.

Let $M$ be a lightlike submanifold of a semi-Riemannian product manifold $(\widetilde{M}, \widetilde{g})$ with quarter symmetric non-metric connection $\widetilde{D}$. Then the Gauss and Weingarten formulas with respect to $\widetilde{D}$ are given by, respectively,

$$
\begin{gathered}
\widetilde{D}_{X} Y=D_{X} Y+\widetilde{h}(X, Y), X, Y \in \Gamma(T M) \\
\widetilde{D}_{X} N=-\widetilde{A}_{N} X+\widetilde{\nabla}_{X}^{l} N+\widetilde{D}^{s}(X, N), N \in \Gamma(\operatorname{ltr} T M),
\end{gathered}
$$

and

$$
\widetilde{D}_{X} W=-\widetilde{A}_{W} X+\widetilde{\nabla}_{X}^{s} W+\widetilde{D}^{l}(X, W), W \in \Gamma\left(s\left(T M^{\perp}\right)\right)
$$

for any $X, Y \in \Gamma(T M)$, where $D_{X} Y, \widetilde{A}_{N} X, \widetilde{A}_{u} X \in \Gamma(T M)$, and $\widetilde{\nabla}^{l}$ and $\widetilde{\nabla}^{s}$ are linear connections on $l t r M$ and $s\left(T M^{\perp}\right)$, respectively. Both $\widetilde{A}_{N}$ and $\widetilde{A}_{u}$ are linear operators on $\Gamma(T M)$. From (5.1), (5.10), (5.11) and (5.12) we obtain

$$
\begin{array}{r}
D_{X} Y=\nabla_{X} Y+\pi(Y) f X, \\
\widetilde{h}(X, Y)=h(X, Y)+\pi(Y) w X, \\
\widetilde{A}_{N} X=A_{N} X-\pi(N) f X, \\
\widetilde{\nabla}_{X}^{l} N=\nabla_{X}^{l} N+\pi(N) w_{l} X, \\
\widetilde{D}^{s}(X, N)=D^{s}(X, N)+\pi(N) w_{s} X,
\end{array}
$$


and

$$
\begin{array}{r}
\widetilde{A}_{W} X=A_{W} X-\pi(W) f X, \\
\widetilde{\nabla}_{X}^{s} W=\nabla_{X}^{s} W+\pi(W) w_{s} X, \\
\widetilde{D}^{l}(X, W)=D^{l}(X, W)+\pi(W) w_{l} X,
\end{array}
$$

for any $X, Y \in \Gamma(T M)$. In where $w_{s}$ and $w_{l}$ are projections on $\operatorname{ltr} T M$ and $s\left(T M^{\perp}\right)$, respectively. From (2.18) and (5.13) we get

$$
\left(D_{X} g\right)(Y, Z)=g\left(h^{l}(X, Y), Z\right)+g\left(h^{l}(X, Z), Y\right)-\pi(Y) g(f X, Z)-\pi(Z) g(f X, Y),
$$

On the other hand, the torsion tensor of the induced connection $D$ is

$$
T^{D}(X, Y)=\pi(Y) f X-\pi(X) f Y .
$$

From last two equations we have the following proposition.

Proposition 5.2. Let $\left(M, g, S(T M), S\left(T M^{\perp}\right)\right)$ be a lightlike submanifold of a semi-Riemannian product manifold $(\widetilde{M}, \widetilde{g})$ with quarter symmetric non-metric connection $\bar{D}$. Then the induced connection $D$ on the lightlike submanifold $M$ is a quarter-symmetric non-metric connection.

With analysis of (2), we can write

$$
\begin{gathered}
D_{X} P Y=D_{X}^{*} P Y+\widetilde{h}^{*}(X, P Y), X, Y \in \Gamma(T M) \\
D_{X} \xi=-\widetilde{A}_{\xi}^{*} X+\widetilde{\nabla}_{X}^{* t} \xi
\end{gathered}
$$

for any $X, Y \in \Gamma(T M)$, where $D_{X}^{*} P Y, \widetilde{A}_{\xi}^{*} X \in \Gamma(s(T M))$ and $\widetilde{h}^{*}(X, P Y) \in \Gamma(\operatorname{Rad} T M)$. From (2.11), (2.12), (5.13), (5.23) and (5.24) we obtain

$$
\begin{array}{r}
D_{X}^{*} P Y=\nabla_{X}^{*} P Y+\pi(P Y) P f X, \\
\widetilde{h}^{*}(X, P Y)=h^{*}(X, P Y)+\pi(P Y) \sum_{i}^{r} \eta_{i}(f X) \xi_{i}, \\
\widetilde{A}_{\xi}^{*} X=A_{\xi}^{*} X-\pi(\xi) P f X, \\
\widetilde{\nabla}_{X}^{* t} \xi=\nabla_{X}^{* t} \xi+\pi(\xi) \sum_{i}^{r} \eta_{i}(f X) \xi,
\end{array}
$$

where $D_{X}^{*} P Y, \widetilde{A}_{\xi}^{*} X \in \Gamma(S(T M)), \eta_{i}(X)=g\left(X, N_{i}\right)$, and $\left\{\xi_{1}, \ldots, \xi_{r}\right\}$ is basis of $\Gamma(\operatorname{RadTM})$ for $i \in\{1, \ldots, r\}$.

From (5.14), (5.27) and (5.15), (5.26) we have

$$
\begin{aligned}
g\left(\widetilde{h}^{l}(X, P Y), \xi\right) & =g\left(h^{l}(X, P Y), \xi\right)+\pi(Y) g\left(w_{l} X, \xi\right) \\
& =g\left(A_{\xi}^{*} X, P Y\right)+\pi(Y) g\left(w_{l} X, \xi\right) \\
& =g\left(\widetilde{A}_{\xi}^{*} X, P Y\right)+\pi(\xi) g(\operatorname{PfX}, P Y)+\pi(Y) g\left(w_{l} X, \xi\right),
\end{aligned}
$$

and

$$
\begin{aligned}
g\left(\widetilde{h}^{*}(X, P Y), N\right) & =g\left(h^{*}(X, P Y), N\right)+\pi(P Y) \eta(f X) \\
& =g\left(A_{N} X, P Y\right)+\pi(P Y) \eta(f X) \\
& =g\left(\widetilde{A}_{N} X, P Y\right)+\pi(N) g(f X, P Y)+\pi(P Y) \eta(f X),
\end{aligned}
$$

Also, from (5.27) we obtain

$$
\begin{aligned}
g\left(\widetilde{A}_{\xi}^{*} P X, P Y\right) & =g\left(A_{\xi}^{*} P X, P Y\right)-\pi(\xi) g(P f X, P Y) \\
& =g\left(A_{\xi}^{*} P Y, P X\right)-\pi(\xi) g(\operatorname{PfX}, P Y),
\end{aligned}
$$


and

$$
g\left(\widetilde{A}_{\xi}^{*} P Y, P X\right)=g\left(A_{\xi}^{*} P Y, P X\right)-\pi(\xi) g(P f Y, P X),
$$

Then from the last two equation and (5.27), we obtain

$$
g\left(\widetilde{A}_{\xi}^{*} P X, P Y\right)-g\left(\widetilde{A}_{\xi}^{*} P Y, P X\right)=\pi(\xi) g(P f Y, P X)-\pi(\xi) g(P f X, P Y),
$$

and

$$
\widetilde{A}_{\xi}^{*} \xi=-\pi(\xi) f \xi,
$$

Lemma 5.1. Let $\left(M, g, S(T M), S\left(T M^{\perp}\right)\right)$ be a semi-invariant $r$-lightlike submanifold of a semi-Riemannian product manifold $(\widetilde{M}, \widetilde{g})$. Then

$$
g\left(\widetilde{h}^{l}(X, Y), \xi\right)=g\left(A_{\xi}^{*} X, Y\right)=g\left(\widetilde{A}_{\xi}^{*} X, Y\right)+\pi(\xi) g(\operatorname{PfX}, Y), \xi \in \Gamma(\operatorname{RadTM}),
$$

for any $X \in \Gamma(L)$ and $Y \in \Gamma\left(L^{\prime}\right)$

Proof. For any $X \in \Gamma(L)$ and $Y \in \Gamma\left(L^{\prime}\right)$ from (5.14) we get $\widetilde{h}^{l}(X, Y)=h^{l}(X, Y)$. Thus from (5.27) we have

$$
g\left(\widetilde{h}^{l}(X, Y), \xi\right)=g\left(h^{l}(X, Y), \xi\right)=g\left(A_{\xi}^{*}, Y\right)=g\left(\widetilde{A}_{\xi}^{*}, Y\right)+\pi(\xi) g(\operatorname{PfX}, Y) .
$$

From Lemma(5.1) and Teorem(4.1), we have the following Corollary

Corollary 5.1. Let $\left(M, g, S(T M), S\left(T M^{\perp}\right)\right)$ be a semi-invariant r-lightlike submanifold of a semi-Riemannian product manifold $(\widetilde{M}, \widetilde{g})$. The following assertions are equivalent:

(i) $M$ is mixed geodesic.

(ii) $M$ is mixed geodesic with respect to quarter symmetric non-metric connection.

(iii) $A_{\xi}^{*} X$ and $A_{W} X$ have no component in $L_{1}$ and $L_{3}$, for any $X \in \Gamma(L)$ and $Y \in \Gamma\left(L^{\prime}\right)$.

(iv) $A_{F Y} X$ have no component $L_{2}$ and $L_{3}$, for any $X \in \Gamma(L)$ and $Y \in \Gamma\left(L^{\prime}\right)$.

Theorem 5.1. Let $\left(M, g, S(T M), S\left(T M^{\perp}\right)\right)$ be a semi-invariant $r$-lightlike submanifold of a semi-Riemannian product manifold $(\widetilde{M}, \widetilde{g})$. For any $X, Y \in \Gamma(L)$ the following assertions are equivalent:

(i) $M$ is $L$-geodesic with respect to quarter symmetric non-metric connection.

(ii) $A_{\xi}^{*} X \in \Gamma\left(L_{1} \perp L_{3}\right)$ and $g\left(Y, A_{W} X\right)=g\left(Y, D^{l}(X, W)\right)$.

(iii) $\nabla_{X} F Y$ have no component $L_{2}$ and $L_{3}$.

(iv) $M$ is $L-$ geodesic.

Proof. For any $X, Y \in \Gamma(L)$, we know that

$$
\widetilde{h}(X, Y)=h(X, Y) .
$$

Thus from Teorem(4.3) we have the proof.

Theorem 5.2. Let $\left(M, g, S(T M), S\left(T M^{\perp}\right)\right)$ be a semi-invariant $r$-lightlike submanifold of a semi-Riemannian product manifold $(\widetilde{M}, \widetilde{g}) . M$ is $L^{\prime}-$ geodesic with respect to quarter symmetric non-metric connection if and only if

$$
A_{F Y} X=-\pi(Y) X, X, Y \in \Gamma\left(L^{\prime}\right)
$$

Proof. For any $X, Y \in \Gamma\left(L^{\prime}\right)$ and $W \in \Gamma\left(s\left(T M^{\perp}\right)\right)$ we obtain

$$
g(\widetilde{h}(X, Y), W)=-g\left(A_{F Y} X+\pi(Y) X, F W\right),
$$

and

$$
g(\widetilde{h}(X, Y), \xi)=-g\left(A_{F Y} X+\pi(Y) X, F \xi\right)
$$

From the last two equations we get the proof. 
Theorem 5.3. Let $\left(M, g, S(T M), S\left(T M^{\perp}\right)\right)$ be a semi-invariant $r$-lightlike submanifold of a semi-Riemannian product manifold $(\widetilde{M}, \widetilde{g})$. The distribution $L$ is parallel with respect to quarter symmetric non-metric connection $D$ if and only if $L$ is parallel with respect to $\nabla$.

Proof. For any $X, Y \in \Gamma(L)$, since $w X=0$ we have

$$
\widetilde{h}(X, F Y)=h(X, F Y) .
$$

Then from teorem(4.6) proof is completed.

Then from teorem(4.6) we have the following corollary.

Corollary 5.2. Let $\left(M, g, S(T M), S\left(T M^{\perp}\right)\right)$ be a semi-invariant r-lightlike submanifold of a semi-Riemannian product manifold $\widetilde{M}$. Then the following assertions are equivalent:

(i) The distribution $L$ is parallel with respect to quarter symmetric non-metric connection $D$.

(ii) The distribution $L$ is parallel with respect to $\nabla$.

(iii) $h(X, F Y)=0, X, Y \in \Gamma(L)$.

(iv) $\widetilde{h}(X, F Y)=0, X, Y \in \Gamma(L)$.

(v) $\left(\nabla_{X} f\right) Y=0, X, Y \in \Gamma(L)$.

Proposition 5.3. Let $\left(M, g, S(T M), S\left(T M^{\perp}\right)\right)$ be a semi-invariant $r$-lightlike submanifold of a semi-Riemannian product manifold $(\widetilde{M}, \widetilde{g})$. The distribution $L^{\prime}$ is parallel with respect to quarter symmetric non-metric connection if and only if

$$
\widetilde{A}_{F Y} X \in \Gamma\left(L^{\prime}\right)
$$

for any $X, Y \in \Gamma\left(L^{\prime}\right)$

Proof. For any $X, Y \in \Gamma\left(L^{\prime}\right)$, since $f X=0$ and $\widetilde{A}_{F Y} X=A_{F Y} X-\pi(F Y) f X$ we have

$$
\widetilde{A}_{F Y} X=A_{F Y} X
$$

From Proposition(4.3) and Proposition(5.3) we have the following corollary.

Theorem 5.4. Let $\left(M, g, S(T M), S\left(T M^{\perp}\right)\right)$ be a semi-invariant $r$-lightlike submanifold of a semi-Riemannian product manifold $(\widetilde{M}, \widetilde{g})$. The distribution $L$ is integrable with respect to quarter symmetric non-metric connection if and only if

$$
\widetilde{h}(X, F Y)=\widetilde{h}(Y, F X), X, Y \in \Gamma(L) .
$$

Proof. For any $X, Y \in \Gamma(L)$, since $w X=0$ we get $\widetilde{h}(X, Y)=h(X, Y)$. From theorem(4.7) we have the proof.

Then from theorem(4.7) we have the following corollary.

Corollary 5.3. Let $\left(M, g, S(T M), S\left(T M^{\perp}\right)\right)$ be a semi-invariant $r$-lightlike submanifold of a semi-Riemannian product manifold $(\widetilde{M}, \widetilde{g})$. Then the following assertions are equivalent:

(i) The distribution $L$ is integrable with respect to quarter symmetric non-metric connection.

(ii) The distribution $L$ is integrable with respect to $\nabla$.

(iii) $\widetilde{h}(X, F Y)=\widetilde{h}(Y, F X), X, Y \in \Gamma(L)$.

(iv) $h(X, F Y)=h(Y, F X), X, Y \in \Gamma(L)$.

(v) $\left(\nabla_{X} f\right) Y=\left(\nabla_{Y} f\right) X, X, Y \in \Gamma(L)$.

Proposition 5.4. Let $\left(M, g, S(T M), S\left(T M^{\perp}\right)\right)$ be a semi-invariant $r$-lightlike submanifold of a semi-Riemannian product manifold $(\widetilde{M}, \widetilde{g})$. The distribution $L^{\prime}$ is integrable with respect to quarter symmetric non-metric connection if and only if

$$
\widetilde{A}_{F Y} X=\widetilde{A}_{F X} Y, X, Y \in \Gamma\left(L^{\prime}\right) .
$$

Proof. For any $X, Y \in \Gamma\left(L^{\prime}\right)$, we know that $f X=0$. Thus from (5.15) and (5.18) we get $\widetilde{A}_{F Y} X=A_{F Y} X$. From Proposition(4.4) we have the our assertion.

Then from proposition(4.4) we give the following corollary. 
Corollary 5.4. Let $\left(M, g, S(T M), S\left(T M^{\perp}\right)\right)$ be a semi-invariant r-lightlike submanifold of a semi-Riemannian product manifold $(\widetilde{M}, \widetilde{g})$. The following assertions are equivalent:

(i) The distribution $L^{\prime}$ is integrable with respect to quarter symmetric non-metric connection.

(ii) The distribution $L^{\prime}$ is integrable with respect to $\nabla$.

(iii) $\widetilde{A}_{F Y} X=\widetilde{A}_{F X} Y, X, Y \in \Gamma\left(L^{\prime}\right)$.

(iv) $A_{F Y} X=A_{F X} Y, X, Y \in \Gamma\left(L^{\prime}\right)$.

\section{References}

[1] Agashe, N. S. and Chafle, M. R., A semi symetric non-metric connection in a Riemannian manifold. Indian J. Pure Appl. Math. 23, 1992, 399-409

[2] Atceken, M. and Kilic, E. Semi-Invariant Lightlike Submanifolds of a Semi-Riemannian Product Manifold. Kodai Math. J. Vol. 30, No. 3, (2007), pp. 361-378.

[3] Duggal, K. L. and Bejancu, A., Lightlike Submanifolds of Semi-Riemannian Manifolds and Applications. Kluwer Academic Publishers, Dordrecht, 1996.

[4] Duggal, K. L. and Bejancu, A., Lightlike submanifolds of codimension two. Math. J. Toyama Univ., 15(1992), 59-82.

[5] Duggal, K. L. and Sahin, B., Screen Cauchy Riemann lightlike submanifolds. Acta Math. Hungar. 106(1-2) (2005), 137-165

[6] Duggal, K. L. and Sahin, B., Generalized Cauchy Riemann lightlike submanifolds. Acta Math. Hungar., 112(1-2), (2006), 113-136.

[7] Duggal, K. L. and Sahin, B., Lightlike submanifolds of indefinite Sasakian manifolds. Int. J. Math. Math. Sci. 2007, Art ID 57585, 1-21.[162]

[8] Duggal, K. L. and Sahin, B., Contact generalized CR-lightlike submanifolds of Sasakian submanifolds. Acta Math. Hungar. 122, No. 1-2, (2009), 45-58.

[9] Duggal, K. L., Sahin, B., Differential Geometry of Lightlike Submanifolds. Birkhauser Veriag AG Basel-Boston-Berlin (2010).

[10] Golab, S., On semi-symmetric metric and quarter-symmetric linear connections, Tensor 29, 1975, 249-254.

[11] Hayden, H. A., Sub-spaces of a space with torsion. Proceedings of the London Mathematical Society. vol. 34, 1932, 27-50.

[12] Kilic, E. and Sahin, B., Radical Anti-Invariant Lightlike Submanifolds of a Semi-Riemannian Product Manifold. Turkish J. Math. 32, (2008), 429-449.

[13] Kilic, E. and Bahadir, O., Lightlike Hypersurfaces of a Semi-Riemannian Product Manifold and Quarter-Symmetric Nonmetric Connections. Hindawi Publishing Corporation International Journal of Mathematics and Mathematical Sciences Volume 2012, Article ID 178390, 17 pages.

[14] Tripathi, M. M., A new connection in a Riemannian manifold. International Journal of Geo. 1, (2008), 15-24.

[15] Yano, K., On semi-symmetric metric connections. Rev. Roumania Math. Pures. Appl. 15, (1970), 1579-1586.

[16] Yano, K., Kon, M., Structures on Manifolds. World Scientific Publishing Co. Pte, Ltd., 1984

\section{Affiliations}

OǦUZHAN BAHADIR

AdDRESS: K.S.U., Department of Mathematics, Faculty of Arts and Sciences, 46100, Kahramanmaras-Turkey E-MAIL: oguzbaha@gmail.com

EROL KILIÇ

AdDRESS: Inonu University, Department of Mathematics, Faculty of Arts and Sciences, 44280, Malatya-Turkey E-MAIL: erol.kilic@inonu.edu.tr 